\title{
Spatial Modelling Concepts for Controlling COVID-19 Risk in Saudi Arabia
}

\author{
Hassan M. Khormi
}

Location and time are important in controlling diseases. This chapter aims to explain how the Saudi authorities implement GIS concepts in controlling the spatial risks of COVID-19. In Saudi Arabia, the impact of COVID-19 is still limited, as the total number of infections did not exceed 74,795 confirmed cases until 25 May 2020, of which 45,668 (61\% out of the total cases) have recovered. This chapter shows regions with high risk and very low risks as well as spatial disease distribution in SA regions. Ar Riyad (17,656 cases), which includes the capital city of SA, Makkah (29,436), Almadinah (9,751), and Ash Sharqiyah (14,012) are the most impacted regions as they recorded most of the cases with 70,855 (95\%) out of the all confirmed cases. Those spatial information must be presented on a different spatial scale, such as city, district, sub-district and house level. Many mobile-based map applications are developed by the Ministries of Health and Interior to, for example, provide insights about disease distributions, help people to find transport during the times of isolation, and show the locations of services, hospitals, etc. These applications are meant to help monitor the impact of the outbreak, manage it, communicate with the security and health services, and help allocate resources, which will help society and institutions to respond effectively.

\subsection{Introduction}

The incidence of COVID-19 started when the virus moved from animal to human populations [1]. The main mode of transmission is from the respiratory tract via droplets or indirectly via fomites and, to a lesser extent, via aerosols. This disease is considered by the World Health Organization (WHO) to be one of the most important and impactful globally. It has been recognized as the most prevalent viral disease in all places around the world [2]. Associations between the incidences and environmental, meteorological and socioeconomic conditions are not yet clearly understood [3]. That makes it difficult to model its risk spatially using GIS. The transmission patterns of the diseases are sensitive to social factors, such as population density, habits, practices, as well as customs and traditions giving rise to occasions and gatherings. This chapter aims to explain how the Saudi authorities implement GIS concepts in controlling the spatial risks of COVID-19. It also shows the current spatial distribution of the disease in Saudi Arabia (SA), with most infected groups according to their nationality, sex and age. Three key factors stand out when looking at COVID-19: human, space (location) and time. When a person gets infected, there is a person involved, and this determines the diseases one gets infected with. Then there is the location, the position on the earth's surface where the event occurred. This position can be accurately located using GPS. 
The location can be used to extract environmental conditions and meteorological variables of areas related certain human practices and people gathering. Time of infection gives valuable information about when the infected person is active and his historical movements. Together with location, time allows one to develop a spatio-temporal picture of the event, and this aspect is extensively used in public health modelling [4].

\subsection{GIS-based Mapping and Modelling}

When we map or model a disease, we take into consideration that everything is related to everything else but near things are more related than distant things [5]. In other words, a person infected with COVID-19 or environmental and climatological conditions closer to an impacted community or disease occurrence should be more conducive to the survival, reproduction and transmission of the virus than conditions further away. This suggests that a study of the geographical location of pathogens and vectors, host interaction, environmental and climatological variables and proximity to human or animal victims is paramount in understanding disease patterns. Spatial analysis answers questions such as what types of habitat that contain the virus, how far the virus's host travels, what populations live in zones of high or low occurrences and what other regions have conditions similar to those where the hosts are currently found and should so be denoted as high-risk areas.

The accessibility of geo-referenced COVID-19 data is of significance in mapping the disease and linking it to environmental or social risk factors. The geographical spreading and seasonal behavior of the disease, as well as its transmission and abundance, are controlled by environmental (such as land use, land cover, elevation) and climatological variables (such as temperature, rainfall, degree days and humidity). Visual displays of quantitative data, such as cases of infections, on cartographic maps for understanding causes has a long history. The best-known example is that of Dr. John Snow and the cholera deaths of London in the mid-nineteenth century [6]. The visualization of spatial epidemiological data on a background of environmental or climatological layer enables one to discern patterns and correlations. Early disease mapping methods were mainly used for communicable diseases to identify sources of infection, rates of spread and general environmental variables present at those sites [7].

\subsection{The Current Spatial Distribution of COVID-19 in Saudi Arabia (SA)}

In Saudi Arabia, the impact of COVID-19 is still limited, as the total number of infections did not exceed 74,795 confirmed cases until 25 May 2020, of which 45,668 (61\% out of the total cases) have recovered. The numbers indicate that males were more at risk of contracting the disease than females. Since the infection started spreading in the country, between 70 and $80 \%$ out of the total recorded cases were male. This is due to the culture of mobility and interaction between men compared with women and the fact that women are more likely to apply the culture of guarding against disease risks and to adhere to regulations and laws targeted at curtailing diseases. But another important reason is that most cases in the Kingdom were recorded between employees of certain companies. So cases involving non-Saudis are around $55 \%$ to $65 \%$ of the total recorded since the beginning of March 2020.

The majority of company employees in Saudi Arabia are males and are mostly citizens of East and Southeast Asia, Pakistan, Bangladesh, and some Arab countries, such as Egypt and Sudan. Some companies, especially those that did not observe the precautionary measures or who delayed in applying them in their premises, were more likely to see infections among their workers. Also, the 
educational and income levels of these employees are low, which required the health and municipal authorities to intervene and provide accommodation to achieve social distancing and create a better precautionary environment. The government decided to supply healthcare and COVID-19 testing for free to all non-Saudis. These steps have a significant impact on reducing the infection rate among workers.

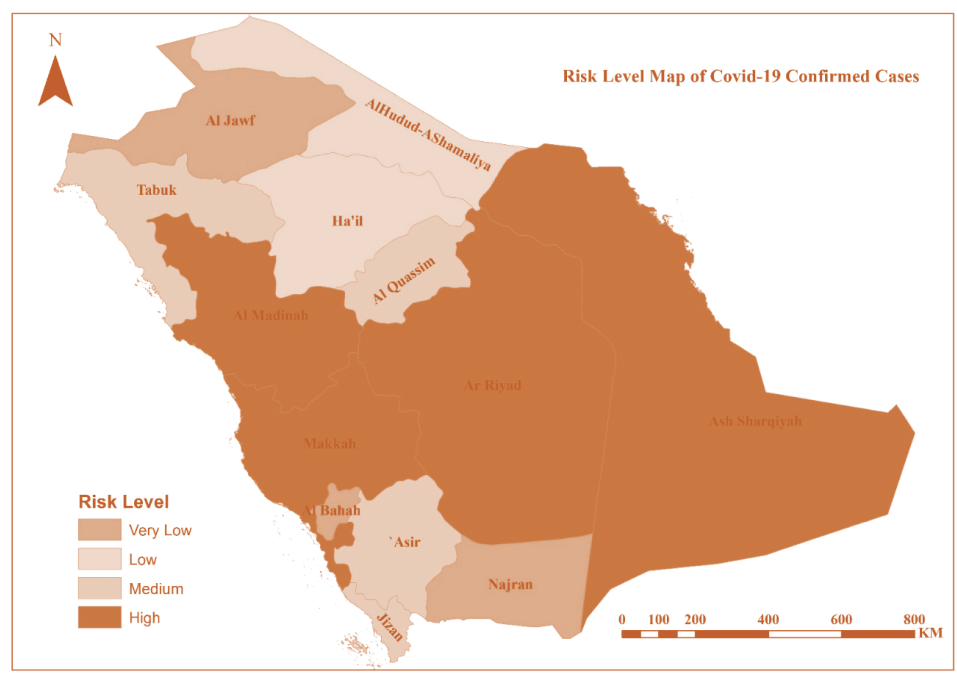

\section{FIGURE 43.1}

COVID-19 risk level and spatial distribution in SA regions (Data Source: Saudi Arabia Health Ministry)

Figure 43.1 shows regions with high risk and very low risks as well as spatial disease distribution in SA regions. Ar Riyad (17,656 cases), which includes the capital city of SA, Makkah $(29,436)$, Almadinah $(9,751)$, and Ash Sharqiyah $(14,012)$ are the most impacted regions as they recorded most of the cases with $70,855(95 \%)$ out of the all confirmed cases. The very low-risk areas are regions that recorded less than 150 cases. Those regions are Al Jawf (84 cases), Najran (147), and Al Baha (148). These numbers show the limited impact of COVID-19 across Saudi Arabia compared with other countries that registered similar numbers daily.

In SA, the deaths have not exceeded 400 since the emergence of the disease. The Makkah region obtained the highest rate of the deaths with $75 \%$, followed by Almadinah (44 deaths), Ar Riyad (24), and Ash Sharqiyah (22). Most of the deaths were related to cases of people with underlying chronic medical conditions, such as diabetes, kidney failure, chronic lung disease, and deficient immune systems. Other regions recorded between zero and four cases of death. Once again, these figures show the minimal impact of the disease in Saudi Arabia compared with other countries around the world that recorded thousands of deaths in one day.

In general, the reason behind the limited negative impact of the disease in Saudi Arabia is the early measures taken by the authorities. That started with the formation of a higher committee headed by the Minister of Health and with the membership including a number of educational, research, service and security sector personnel to review international events related to the disease and assess the current conditions, make reference comparisons and gather experiences to address the disease, especially from countries that had already been ravaged by it.

At that time, the Saudi authorities began gradually isolating a number of the most affected neighbourhoods, then cities. Then they began closing international entry points and finally proceeded to completely isolating all Saudi regions. In all those steps and actions taken by the authorities, the geographical concepts were present, starting from determining the geographical location of the infected person, the relatives and friends of that person, the geographical locations of their movements, determining the boundaries of the closed neighbourhoods, setting a map to be 
used by the public, and determining on the maps the ports to be used for entering and exiting the isolated areas.

There is high awareness as to the use of GIS in all aspects of disease surveillance and system control. The Saudi Health Ministry developed many mobile applications and a GIS platform for uploading and illustrating locations and data of, for example, confirmed, active and recovered cases based on small and large geographical scales to present the events on house (point) (Figure 43.2A), sub-district, district, county, region and country (Figure 43.2B).
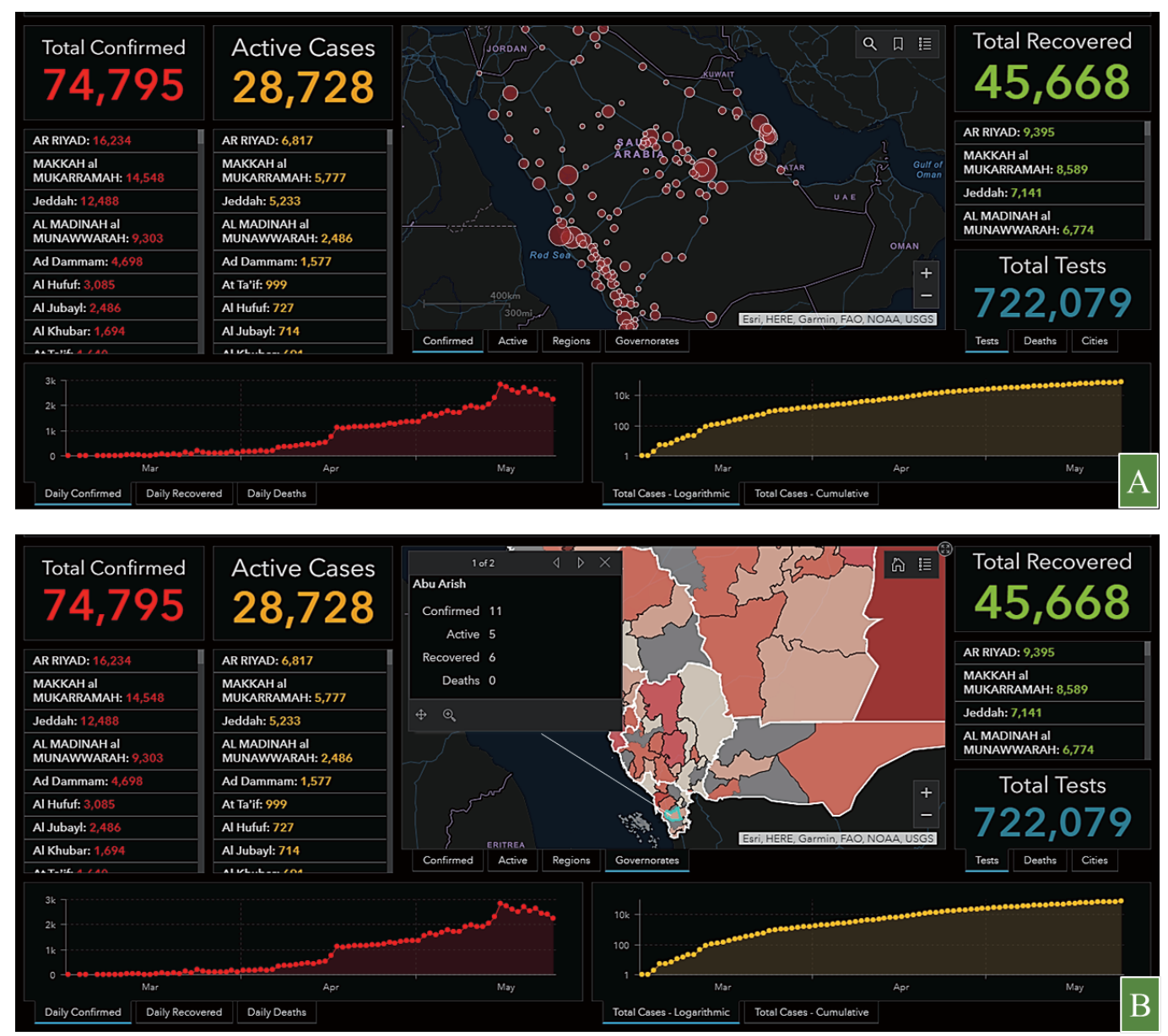

\section{FIGURE 43.2}

Interface of GIS platform developed by the Health Ministry to show daily spatial distribution and information of COVID-19 (https://covid19.moh.gov.sa/)

This GIS-based system can go far beyond the early studies on establishing correlates. So the main purpose can be enhanced to describe the geographical differences in disease occurrence for formulating aetiological hypotheses. Also, the system locates unusual high-risk hotspots to develop a preventive plan. Another purpose is to improve the reliability of disease risk models for allocating resources. Moreover, the system can undertake sophisticated spatial analyses of environmental features and disease rates, together with geostatistical analysis to statistically verify associations $[8-10]$. 


\subsection{Conclusion}

The spatial information must be presented on a different spatial scale, such as city, district, sub-district and house level. Many mobile-based map applications are developed by the Ministries of Health and Interior to, for example, provide insights about disease distributions, help people to find transport during the times of isolation, and show the locations of services, hospitals, etc. The main purpose of these applications is to help monitor the impact of the outbreak, manage it, communicate with the security and health services, and help allocate resources, which will help society and institutions to respond effectively. Accordingly, we summarize the aforementioned in this scientific paper to avoid the effects of the COVID-19 epidemic quickly. Immediate plans should be developed for the danger areas in your environment through several steps relying on GIS. The most important of these steps is to map the confirmed and active cases, deaths and retrieval operations to determine the whereabouts of the COVID-19 infections and to update the records continuously. Mapping the spatiotemporal distribution of the disease can reveal how the infection has spread over time and where you might want to target the interventions. Publishing a map of at-risk populations will provide information about how COVID-19 will unevenly affect some demographics, such as the elderly and those with chronic diseases as well as non-Saudi workers. Mapping social vulnerability, age and other factors also helps to monitor groups and areas at risk. It is important to map national capabilities and facility locations, such as a map of facilities, health providers, medical resources, equipment, goods and other services to understand and respond to the current and potential impacts of COVID-19. Interactive web maps, dashboard apps, story maps and historical tracking can be used to help quickly communicate with people about the national situation so everyone will be aware of all the procedures.

\section{References}

[1] Mackenzie, J. S., Smith, D. W. (2020) COVID-19: a novel zoonotic disease caused by a coronavirus from China: what we know and what we don't [published online ahead of print, 2020 Mar 17]. Microbiol Aust. 2020;MA20013. doi:10.1071/MA20013.

[2] WHO/2019-nCoV/Sci_Brief/Transmission_modes/2020.2

[3] Briz-Redón, Á., Serrano-Aroca, Á. (2020) A spatio-temporal analysis for exploring the effect of temperature on COVID-19 early evolution in Spain. Science of the Total Environment, Volume 728.

[4] Khormi, H., Kumar, L. (2015) Modeling interactions between vector-borne diseases and environment using GIS. CRC Press, Taylor and Fancies.

[5] Tobler, W. (1970) A computer movie simulating urban growth in the Detroit region". Economic Geography, 46(2): 234-240.

[6] Snow, J. (1855) On the mode of communication of cholera. London: John Churchill (2nd ed).

[7] Howe, G. M. (1989) Historical evolution of disease mapping in general and specifically of cancer mapping. Recent Results Cancer Research, 114: 1-21.

[8] Rytkonen, M. J. P. (2004) Not all maps are equal: GIS and spatial analysis in epidemiology. International Journal of Circumpolar Health, 63(1): 9-24.

[9] Lawson, A. B., Biggeri, A. B., Boehning, D., Lesaffre, E., Viel, J. F., Clark, A., Schlattmann, P., Divino, F. (2000) Disease mapping models: an empirical evaluation. Disease Mapping Collaborative Group. Stat Med, 19: $2217-2241$.

[10] Lawson, A. B., Böhning, D., Biggeri, A., Lesaffre, E., Viel, J. F. (1999) Disease mapping and its uses. In: Lawson, A., Biggeri, A., Böhning, D., Lesaffre, E., Viel, J. F. and Bertollini, R. (eds.), Disease mapping and risk assessment for public health. West Sussex: John Wiley \& Sons Ltd; 3-13. 
$\Longrightarrow$ Taylor \& Francis Taylor \& Francis Group

http://taylorandfrancis.com 